\title{
Health Care Utilization and Economic Burden in Patients with Central Precocious Puberty: An Assessment of the Commercially Insured and Medicaid Populations
}

\author{
Karen Klein, MD; Ahmed M. Soliman, MS, PhD; Machaon Bonafede, PhD, MPH; \\ James K. Nelson, PhD; and Elizabeth Grubb, PhD, MBA
}

\begin{abstract}
BACKGROUND: Central precocious puberty (CPP), early onset of puberty caused by the premature activation of the hypothalamic-pituitary-gonadal axis, is a rare disease affecting children of both sexes. There is limited evidence that quantifies the economic burden of CPP.

OBJECTIVE: To characterize the health care resource utilization (HRU) and costs among patients with CPP who were treated with gonadotropinreleasing hormone (GnRH) agonists, for those insured commercially and with Medicaid.

METHODS: Eligible CPP patients for this retrospective cohort analysis were aged $\leq 12$ years; were diagnosed between January 1, 2010, and September 30, 2014; and had at least 1 prescription for an FDA-approved GnRH agonist: leuprolide or histrelin (first prescription = index date). CPP patients had to be continuously enrolled in the MarketScan Commercial or Medicaid Database for at least 12 months before and after the index date. Control patients were randomly selected from all eligible non-CPP patients and $\mathrm{N}: 1$ matched on demographic characteristics with up to 20 controls per case. Clinical comorbidities, HRU, and costs were compared between study cohorts. Health care costs were examined via multivariable analysis to adjust for baseline differences between patients and controls. Treatment patterns among CPP patients were also characterized.
\end{abstract}

RESULTS: There were 1,236 CPP patients and 24,206 controls with commercial insurance and 673 CPP patients and 11,965 controls with Medicaid insurance who met the inclusion criteria. Across payers, the mean age of CPP patients ranged from 7.6 years (Medicaid) to 8.5 (commercial), and $80 \%-87 \%$ were female. The mean observed duration (SD) of treatment with any approved GnRH agonist was $1.51(0.98)$ years for commercial patients and 1.22 (1.04) for Medicaid patients. The mean age of discontinuation among patients who ceased GnRH agonist treatment ranged from 8.7 to 9.6 years. In the first year post-index, CPP patients had a greater number of unique diagnosis codes, unique medications, and comorbid conditions than controls. They also had significantly higher all-cause and diseasemonitoring related HRU. After adjusting for baseline characteristics, CPP patients with Medicaid insurance spent 6.42 times more $(\$ 16,768$ [\$31,460] vs. $\$ 2,610$ [\$4,897]), and patients with commercial insurance spent 12.25 times more $(\$ 19,940[\$ 20,132]$ vs. $\$ 1,628[\$ 1,645])$ on health care in the year following treatment initiation than matched controls.

CONCLUSIONS: Patients with CPP have substantially more comorbidities and greater HRU and costs than their non-CPP peers.

J Manag Care Spec Pharm. 2019;25(7):836-46

Copyright $\odot 2019$, Academy of Managed Care Pharmacy. All rights reserved.

\section{What is already known about this subject}

Central precocious puberty (CPP) is a rare disease in which sexual development starts before age 8 in girls or age 9 in boys. The primary complications of CPP include early puberty, early menstrual periods, and short stature from early bone maturation, but other long-term risks are being studied in the areas of psychological impact, polycystic ovary syndrome, and long-term cardiovascular risks.

Standard care involves treatment with long-acting gonadotropinreleasing hormone agonists and disease-monitoring laboratory and radiographic tests.

\section{What this study adds}

This retrospective, observational cohort study compared the comorbidity burden, health care resource utilization (HRU), and costs of children aged $\leq 12$ years diagnosed and treated for CPP with either U.S. commercial or Medicaid insurance to matched controls.

Patients with CPP had higher comorbidity burden and HRU than matched controls at baseline, and Medicaid patients had a higher comorbidity burden than patients with commercial insurance.

In the year following treatment initiation, patients with CPP spent 6-12 times as much on health care compared with matched controls, due largely to spending on outpatient services and outpatient pharmacy claims.

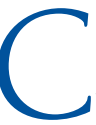
entral precocious puberty (CPP) is the early onset of puberty (before 8 years in girls and 9 years in boys) because of the premature activation of the hypothalamicpituitary-gonadal axis. ${ }^{1}$ CPP is roughly 10 times more common in girls, and the prevalence has been estimated to be as low as 1 in 10,000 and as high as 1 in 1,000.2,3 These numbers are confounded by the variety of methods employed to verify the diagnosis, and the separate secular trend of decreasing average age of puberty. ${ }^{4,5}$

Although the majority of CPP cases are idiopathic, cranial magnetic resonance imaging is recommended in all cases, as the presence of intracranial lesions, even in the absence of neurological symptoms, has been reported as 11\%-31\% in girls 
and 33\%-90\% in boys. ${ }^{3,6-8}$ International adoption has also been associated with increased risk of CPP, as have certain genetic factors, including activation of kisspeptin and its cognate receptor or the inactivation of the makorin ring finger protein 3 (MKRN3) gene. ${ }^{3,9}$ In addition, long-term exposure to and sudden withdrawal of sex steroids can result in secondary central precocious puberty. ${ }^{10}$

Untreated, CPP results in short stature from early bone maturation and closure of the bone epiphyses. ${ }^{11}$ In addition, early menarche has been associated with higher rates of substance abuse, polycystic ovary syndrome, obesity, longterm cardiovascular risks, depression, and other psychosocial issues. ${ }^{12-16}$ The gold-standard treatment for CPP is the regression or stabilization of pubertal symptoms through treatment with long-acting gonadotropin-releasing hormone ( $\mathrm{GnRH})$ agonists. ${ }^{8,17}$ The current GnRH agonist treatments approved by the U.S. Food and Drug Administration for CPP are leuprolide acetate or triptorelin injections and histrelin acetate-releasing implants. ${ }^{18,19}$

In the short term, GnRH agonist treatment can delay puberty and help patients achieve their target final height. ${ }^{20}$ In the long term, previous studies have shown that girls treated for idiopathic CPP are no more likely than their non-CPP peers to have an increased risk of obesity, metabolic derangements, cancer, or reproductive challenges in their third and fifth decade. ${ }^{21,22}$ The same is true for girls with untreated CPP, except in 1 study in adulthood that suggests these women are more likely to suffer from clinical hyperandrogenism and fertility problems than their treated or non-CPP peers. ${ }^{22}$ Little is known about the long-term effects of CPP on males because of its low prevalence and the high incidence of concomitant central nervous system lesions.

Although the literature on the economic cost of CPP is limited to a single study of 172 commercially insured patients, there is reason to suspect that CPP is associated with a significant economic burden. ${ }^{23}$ First, in addition to the GnRH agonist treatment costs, effective treatment of $\mathrm{CPP}$ requires periodic laboratory tests to confirm hormone suppression and radiographic screening to track bone age. ${ }^{24,25}$ Second, previous analysis of real-world data has suggested that patients with CPP have more comorbid conditions than their non-CPP peers leading to greater all-cause health care utilization..$^{26}$ The current retrospective matched cohort study used administrative claims data to expand on the existing research on health care resource utilization (HRU) and costs among patients with CPP compared with their non-CPP peers.

\section{Methods}

\section{Data Source}

This retrospective cohort study used deidentified, patientlevel administrative claims data from the IBM Watson Health MarketScan Commercial Database (commercial) and
MarketScan Multistate Medicaid Database (Medicaid) for the period between January 1, 2009, and September 30, 2015. The commercial database contains the data for approximately 40 million employees and their dependents annually under a variety of fee-for-service and managed care health plans across the United States. The Medicaid database covers over 44 million enrollees pooled from approximately 12 geographically dispersed state Medicaid programs. Each database captures the inpatient medical, outpatient medical, and outpatient prescription drug data for its respective covered population, and together form a nationally representative sample. All study data were obtained using International Classification of Diseases, Ninth Revision, Clinical Modification (ICD-9-CM) codes; Current Procedural Terminology, 4th Edition, codes; Healthcare Common Procedure Coding System codes (HCPCS); and National Drug Code (NDC) numbers.

All database records are statistically deidentified and certified to be fully compliant with U.S. patient confidentiality requirements set forth in the Health Insurance Portability and Accountability Act (HIPAA). Because this study used only deidentified patient records and did not involve the collection, use, or transmittal of individually identifiable data, institutional review board approval to conduct this study was not necessary.

\section{Patient Selection and Cohort Matching}

Patients in each database with at least 1 nondiagnostic claim with the ICD-9-CM code 259.1 in any diagnosis position between January 1, 2010, and September 30, 2014, were identified. The ICD-9-CM code 259.1 is not exclusive to patients with CPP, so patients were also required to have at least 1 claim on or after their first CPP diagnosis for a GnRH agonist approved for treatment of CPP (histrelin or leuprolide). The index date was the date of the first claim for a GnRH agonist in the study period following CPP diagnosis. Patients were required to have continuous enrollment in medical and pharmacy benefits for a minimum of 12 months before and after the index date. Patients were excluded if they used a GnRH agonist in the 12-month pre-index period or if they were aged more than 12 years at the index date.

Non-CPP controls were identified in each database and matched 1 to 1 with CPP patients within their respective database based on baseline demographic characteristics, including age, sex, plan type, region of residence, and race (Medicaid patients only). Up to 20 controls were matched per CPP patient. The index date for controls was randomly assigned to match the year-by-year distribution of the CPP cohort. For inclusion in the study, controls had to be aged $\leq 12$ years at the index date and have continuous enrollment in medical and pharmacy benefits for a minimum of 12 months before and after the index date. There could also be no evidence of CPP-related ICD-9-CM codes or use of any GnRH agonists 
Age in years, mean $(\mathrm{SD})$

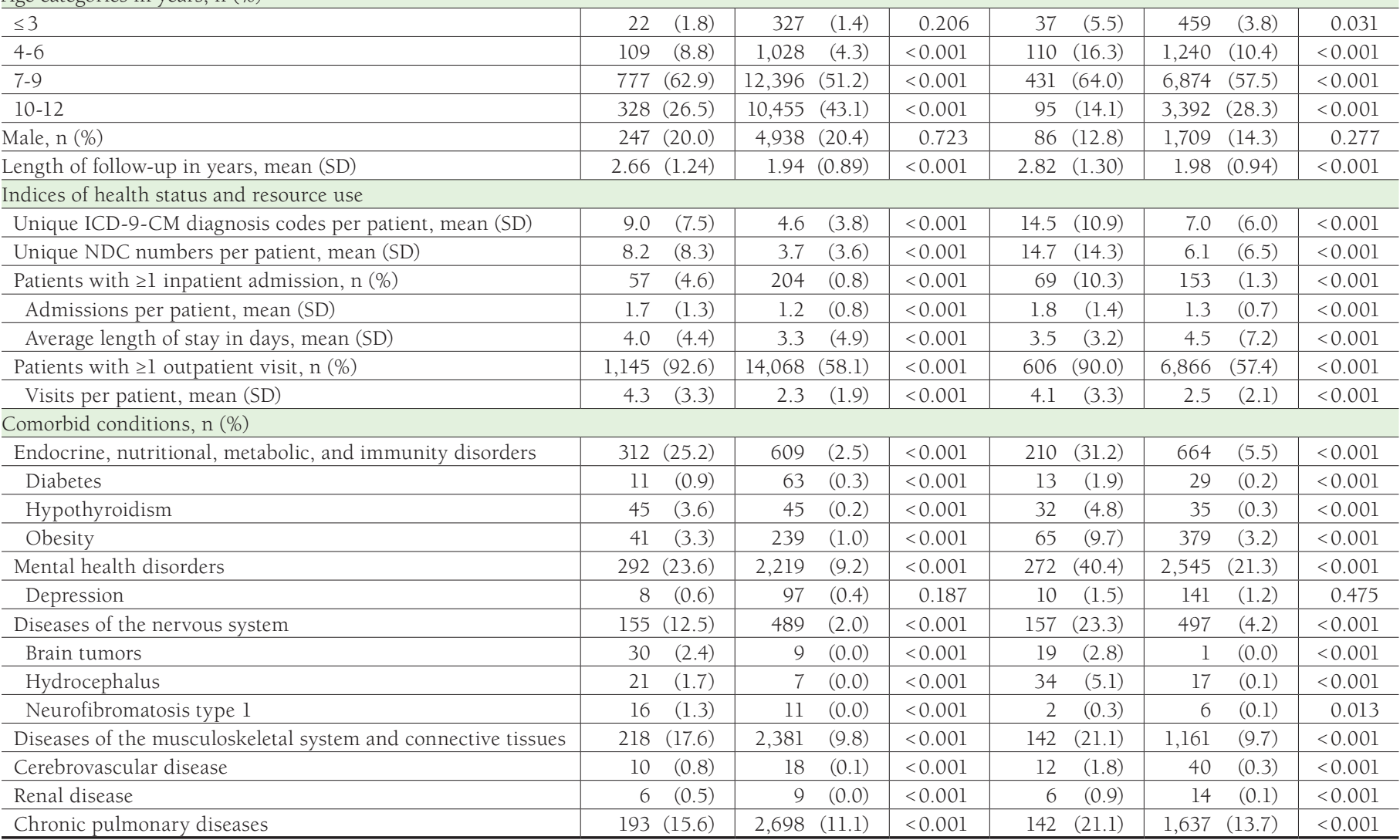

aStatistical tests compared case to controls within each insurance type.

$C P P=$ central precocious puberty; ICD-9-CM=International Classification of Diseases, Ninth Revision, Clinical Modification; NDC=National Drug Code; $S D=$ standard deviation.

in their entire claims history through the 12-month post-index period. No other exclusion criteria were applied.

\section{Baseline Characteristics}

Patient demographics, including age, sex, type of insurance plan, index year, geographic region (commercial only), urban or rural residency, and race (Medicaid only) were measured on the index date. Urban or rural residence classification was based on whether the primary subscriber's address was located within a metropolitan statistical area. Available post-index follow-up data were measured starting at the index date and ending at a record of inpatient death, the end of continuous enrollment, or the end of the study period, whichever occurred first.

Clinical characteristics were measured during the 12-month pre-index period and included indices of general health status and resource use, as well as the presence of certain comorbid conditions. The indices were the number of ICD9-CM diagnosis codes per patient, the number of unique NDC numbers per patient, the number of patients with at least 1 inpatient admission, the number of inpatient admissions per patient, the length of inpatient stay, the number of patients with at least 1 outpatient office visit, and the number of visits per patient. Comorbid conditions were measured across 7 clinical categories: (1) endocrine, nutritional, metabolic, and immunity disorders; (2) mental health disorders; (3) diseases of the nervous system; (4) diseases of the musculoskeletal system; (5) cerebrovascular diseases; (6) renal diseases; and (7) chronic pulmonary diseases. These general categories were modeled after the approach of Chen et al. (2012). ${ }^{23}$ In addition, the presence of 7 specific comorbid conditions was 


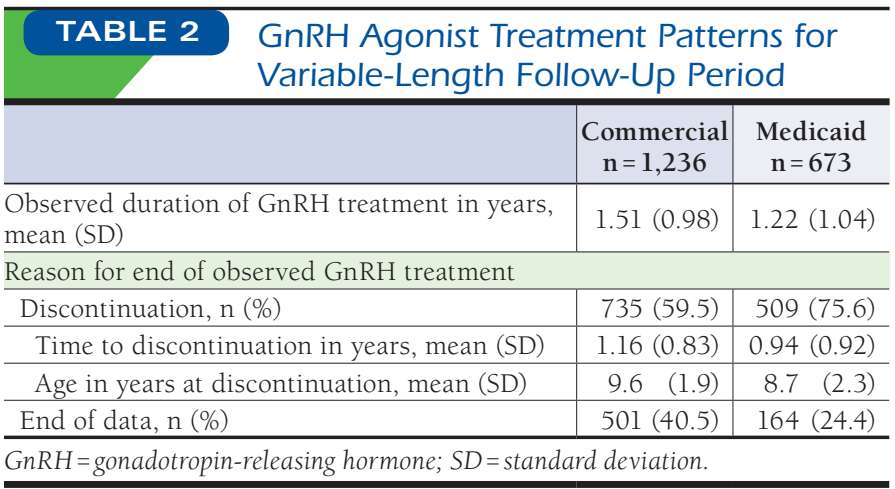

also recorded: diabetes, hypothyroidism, obesity, depression, brain tumors, hydrocephalus, and neurofibromatosis type 1 . The ICD-9-CM codes used to identify comorbid conditions are listed in Appendix A (available in online article).

\section{Outcomes}

The outcomes evaluated were GnRH agonist treatment patterns, all-cause and disease-monitoring related HRU and all-cause health care costs. GnRH agonist treatment patterns including duration and reason for the end of observed treatment (either treatment discontinuation or end of data) were recorded. The entire length of available follow-up data for each patient was used to assess treatment patterns. Discontinuation was defined as a gap of 120 days in treatment (on-label treatment with leuprolide may be every 90 days) with no additional evidence of initiation of any GnRH agonist. For leuprolide, the date of discontinuation was determined by the days of supply for the final pharmacy claim, or 30 or 90 days following the final injection of the drug, depending on the formulation (for injections with costs of $<\$ 1,000$, a 30 -day formulation was assumed; for injections with costs of $\geq \$ 1,000$, a 90 -day formulation was assumed). ${ }^{23}$ For histrelin implants, the time of discontinuation was defined as the shortest of 12 months following the last implant, or the time from an initial claim to a second claim for a HCPCS code specifying implant removal without replacement or initiation of leuprolide. Time to discontinuation and age at discontinuation were recorded.

All-cause HRU including inpatient admissions, outpatient office visits, and outpatient prescriptions was measured for the 12-month post-index period. HRU associated with CPP treatment monitoring —including pediatrician endocrinology visits, laboratory panels, imaging procedures, and stimulation tests (corticotropic or GnRH) —were also described.

All-cause costs including inpatient admissions, outpatient office visits, laboratory outpatient services, radiology outpatient services, outpatient prescriptions, and other outpatient services were measured during both the 12 -month pre-index and 12-month post-index periods. Costs for all-cause HRU were calculated based on the paid amounts for adjudicated claims including portions paid by both insurers and the patient. All costs are reported as per person per year and were adjusted to 2015 U.S. dollars using the medical care component of the Consumer Price Index. ${ }^{27}$ Costs for services provided under capitated arrangements were estimated using payment proxies computed based on paid claims at the procedure level.

\section{Statistical Analysis}

Bivariate statistics were used to compare all baseline characteristics and outcome variables between the treated CPP cohort and matched control cohort. For continuous variables, means and standard deviations were reported, and 2-tailed Student's t-tests were used to test for significant differences. For categorical variables, counts and percentages were reported, and chisquare tests were used for significance testing. The alpha level for all statistical tests was 0.05 .

Multivariable analysis, using generalized linear models with a gamma distribution and log-link function, was employed to evaluate the incremental health care costs associated with a CPP diagnosis while adjusting for covariates. Separate models were built for commercial and Medicaid-insured populations. Covariates included were age, sex, baseline total health care costs, baseline NDC count, index year, insurance plan type, geographic region (commercial only), race (Medicaid only), and a subset of baseline clinical characteristics (brain tumors, depression, diabetes, hydrocephalus, hypothyroidism, mental health disorders, musculoskeletal disorders, nervous system disorders, neurofibromatosis, and obesity).

\section{Results}

\section{Patient Characteristics}

There were 80,727 commercially insured and 35,542 Medicaidinsured patients with at least 1 nondiagnostic claim with a diagnosis of precocious puberty. Of these, 1,236 and 673, respectively, met all the selection criteria for GnRH agonisttreated CPP patients (see Appendix B for the detailed attrition diagram for patient selection, available in online article). These CPP patients were matched N:1 to 24,206 commercially insured and 11,965 Medicaid-insured non-CPP controls.

The average age (standard deviation [SD]) of commercially insured CPP patients was 8.5 (1.9) years with $62.9 \%$ of patients aged between 7 and 9 years (Table 1). Among Medicaidinsured CPP patients, the average age (SD) was 7.6 (2.1) years, and $64.0 \%$ were aged between 7 and 9 years at treatment initiation. The ratio of female to male CPP patients was 4:1 among commercially insured patients and almost 7:1 among Medicaid-insured patients. The average length of available covered enrollment after the index date was over 2.5 years in both groups.

Across commercially insured and Medicaid populations, CPP patients had more comorbidities and greater HRU in the 
TABLE 3 All-Cause and Disease-Monitoring Related Health Care Resource Utilization in the 12-Month Post-Index Period

\begin{tabular}{|c|c|c|c|c|c|c|c|}
\hline & \multicolumn{3}{|c|}{ Commercial } & \multicolumn{3}{|c|}{ Medicaid } & \multirow{2}{*}{$\begin{array}{c}\begin{array}{c}\text { Commercial } \\
\text { vs. Medicaid }\end{array} \\
\text { P Value } \\
\end{array}$} \\
\hline & $\begin{array}{c}\text { CPP } \\
\mathrm{n}=1,236\end{array}$ & $\begin{array}{c}\text { Controls } \\
\mathbf{n}=24,206\end{array}$ & $P$ Value ${ }^{\mathrm{a}}$ & $\begin{array}{c}\text { CPP } \\
\mathbf{n}=673\end{array}$ & $\begin{array}{c}\text { Controls } \\
\mathrm{n}=11,965\end{array}$ & $P$ Value ${ }^{a}$ & \\
\hline \multicolumn{8}{|l|}{ All-cause health care utilization } \\
\hline Patients with $\geq 1$ inpatient admission, $\mathrm{n}(\%)$ & $63(5.1)$ & $197(0.8)$ & $<0.001$ & $(7.6)$ & $182(1.5)$ & $<0.001$ & 0.029 \\
\hline Admissions per patient, mean (SD) & $1.5 \quad(1.0)$ & $1.3 \quad(0.9)$ & $<0.001$ & $(1.4)$ & $1.5 \quad(1.3)$ & $<0.001$ & $<0.001$ \\
\hline Length of stay per admission in days, mean (SD) & $4.3(6.3)$ & $3.5(3.2)$ & $<0.001$ & $3.8 \quad(4.8)$ & $6.6(20.2)$ & $<0.001$ & 0.043 \\
\hline Patients with $\geq 1$ outpatient visit, $\mathrm{n}(\%)$ & $1,125(91.0)$ & $13,841(57.2)$ & $<0.001$ & $622(92.4)$ & $6,546(54.7)$ & $<0.001$ & 0.293 \\
\hline Visits per patient, mean (SD) & $4.5(3.7)$ & $2.2(1.9)$ & $<0.001$ & $4.9 \quad(3.7)$ & $2.5(2.3)$ & $<0.001$ & 0.049 \\
\hline Patients with $\geq 1$ outpatient prescription claim, n (\%) & $1,114(90.1)$ & $14,088(58.2)$ & $<0.001$ & $639(94.9)$ & $7,778(65.0)$ & $<0.001$ & $<0.001$ \\
\hline Prescriptions per patient, mean (SD) & $14.3(15.5)$ & $4.8 \quad(6.5)$ & $<0.001$ & $25.4(29.5)$ & $8.7(12.8)$ & $<0.001$ & $<0.001$ \\
\hline \multicolumn{8}{|l|}{ Disease-monitoring related utilization } \\
\hline Pediatric endocrinology visits, n (\%) & $588(47.6)$ & $127 \quad(0.5)$ & $<0.001$ & $69(10.3)$ & $20 \quad(0.2)$ & $<0.001$ & $<0.001$ \\
\hline Visits per patient, mean (SD) & $6.0 \quad(7.4)$ & $3.4 \quad(3.3)$ & $<0.001$ & $2.7 \quad(2.1)$ & $4.1 \quad(6.5)$ & $<0.001$ & $<0.001$ \\
\hline Laboratory panels, ${ }^{\mathrm{c}} \mathrm{n}(\%)$ & $736(59.5)$ & $41 \quad(0.2)$ & $<0.001$ & $354(52.6)$ & $33(0.3)$ & $<0.001$ & 0.003 \\
\hline Laboratory panels per patient, mean (SD) & $2.4 \quad(2.3)$ & $2.0 \quad(1.2)$ & $<0.001$ & $2.0 \quad(1.5)$ & $2.2(1.6)$ & $<0.001$ & $<0.001$ \\
\hline Imaging procedures, $\mathrm{n}(\%)$ & $196(15.9)$ & $143 \quad(0.6)$ & $<0.001$ & $147(21.8)$ & $88 \quad(0.7)$ & $<0.001$ & 0.001 \\
\hline Imaging procedures per patient, mean (SD) & $2.3(2.2)$ & $1.6 \quad(1.0)$ & $<0.001$ & $2.4 \quad(1.7)$ & $1.8(0.7)$ & $<0.001$ & 0.818 \\
\hline Stimulation tests, n (\%) & $14 \quad(1.1)$ & $0 \quad(0.0)$ & $<0.001$ & $(0.3)$ & $0 \quad(0.0)$ & $<0.001$ & 0.056 \\
\hline Tests per patient, mean (SD) & $1.3(0.5)$ & $0 \quad(0.0)$ & $<0.001$ & $(0.0)$ & $0 \quad(0.0)$ & N/A & $<0.001$ \\
\hline
\end{tabular}

aStatistical tests compared case to controls within each insurance type.

bStatistical tests compared commercial to Medicaid cases with CPP.

'Includes panels that test for sex steroids.

$C P P=$ central precocious puberty; $N / A=$ not applicable; $S D=$ standard deviation .

pre-index period than the matched controls (Table 1). CPP patients had a significantly higher disease burden (SD), as measured by unique ICD-9-CM diagnostic codes (commercial: 9.0 [7.5] CPP vs. 4.6 [3.8] controls, $P<0.001$; Medicaid: 14.5 [10.9] CPP vs. 7.0 [6.0] controls, $P<0.001$ ) or by unique NDC numbers (commercial: 8.2 [6.0] CPP vs. 3.7 [3.6] controls, $P<0.001$; Medicaid: 14.7 [14.3] CPP vs. 6.1 [6.5] controls, $P<0.001)$ in their claims record. Commercially insured CPP patients were 5 times more likely and Medicaid-insured CPP patients were 7 times more likely than controls to have at least 1 inpatient admission in the pre-index period $(P<0.001$; Table 1).

All evaluated comorbid conditions were more common in the CPP cohorts than the matched controls (Table 1) with the exception of depression, which was rare and was not statistically different between the CPP and non-CPP cohorts (commercial: $0.6 \%$ CPP vs. $0.4 \%$ controls, $P=0.187$; Medicaid: $1.5 \%$ CPP vs. $1.2 \%$ controls, $P=0.475)$. Disorders that fell into the category of endocrine, nutritional, metabolic, or immunity were 5-10 times more common in the CPP cohort than the controls, and diseases of the nervous system were 5-6 times more common in the CPP cohort $(P<0.001)$. Notably, all comorbid conditions with a prevalence of at least $1 \%$ were more common among Medicaid-insured CPP patients than commercially insured CPP patients (Table 1).

\section{GnRH Agonist Treatment Patterns}

GnRH agonist treatment patterns for the variable follow-up period are reported in Table 2 . The average observed duration (SD) of GnRH agonist treatment was 1.51 (0.98) years for commercially insured patients and 1.22 (1.04) years for Medicaid-insured patients. GnRH agonist discontinuation was captured for over 59\% of commercially insured patients and $75 \%$ of Medicaid-insured patients before the end of their follow-up period. Among these patients with an observed discontinuation, the time to discontinuation (SD) of treatment was 1.16 (0.83) years and 0.94 (0.92) years, respectively. Commercially insured patients were slightly older when they discontinued therapy (9.6 [1.9] years commercial vs. 8.7 [2.3] years Medicaid). If a patient switched from commercial insurance to Medicaid, or vice versa, their data would look like a discontinuation of treatment when it was not, so caution is needed in interpretation of age at discontinuation and duration of treatment.

\section{Health Care Resource Utilization}

All-cause and disease-monitoring related HRU, by service category, for the first 12 -months post-index is reported in Table 3. Regardless of the payer type, CPP patients had higher utilization in every category compared with matched controls $(P<0.001)$. CPP patients were 5-6 times more likely than 
Health Care Utilization and Economic Burden in Patients with Central Precocious Puberty: An Assessment of the Commercially Insured and Medicaid Populations

\begin{tabular}{l} 
TABLE 4 All-Cause Health Care Expenditures for the 12 Months Pre- and Post-Index \\
Date Reported as per Person per Year \\
\hline
\end{tabular}

astatistical tests compared case to controls within each insurance type.

$C P P=$ central precocious puberty; $S D=$ standard deviation .

controls to have at least 1 inpatient admission in the 12-month post-index period (commercial: $5.1 \% \mathrm{CPP}$ vs. $0.8 \%$ controls, $P<0.001$; Medicaid: $7.6 \%$ CPP vs. $1.5 \%$ controls, $P<0.001$ ). Over $90 \%$ of CPP patients had at least 1 outpatient office visit, and $90.1 \%-94.9 \%$ had at least 1 outpatient prescription claim, while controls had 54.7\%-57.2\% and 58.2\%-65.0\% utilization rates, respectively.

Over the first year following the index date, $47.6 \%$ of commercially insured CPP patients, but only $10.3 \%$ of Medicaid-insured CPP patients, had at least 1 endocrinology visit and the average number of visits by commercially insured patients was double that of Medicaid-insured patients (Table 3). Usage of laboratory panels to monitor sex steroids was $52.6 \%$ $59.5 \%$ among CPP patients, compared with $<0.5 \%$ among control patients. In addition, $15.9 \%-21.8 \%$ of CPP patients had imaging procedures, and $0.3 \%-1.1 \%$ had GnRH or corticotropic stimulation tests (Table 3).

\section{Costs}

Consistent with the higher observed HRU across all categories, the unadjusted health care costs across all categories were higher for CPP patients than controls $(P<0.001$; Table 4). Compared with controls, patients with CPP had higher total unadjusted health care costs (SD) in the 12-month pre-index period (commercial: $\$ 14,338$ [\$32,157] CPP vs. $\$ 1,595[\$ 9,162]$ controls, $P<0.001$; Medicaid: $\$ 16,097$ [\$37,831] CPP vs. $\$ 2,484$ $[\$ 11,557]$ controls, $P<0.001)$ and in the 12 -month post-index period (commercial: $\$ 37,135[\$ 40,436]$ CPP vs. $\$ 1,665[\$ 9,099]$ controls, $P<0.001$; Medicaid: $\$ 29,249$ [\$50,484] CPP vs. $\$ 2,665$ [ $\$ 14,863]$ controls, $P<0.001$ ).

High costs for CPP patients are derived primarily from outpatient prescriptions costs and other outpatient services costs. After adjusting for baseline costs, demographics, and clinical characteristics, the all-cause health care costs in the year following CPP treatment initiation were 12.3 times higher for commercially insured patients and 6.4 times higher for Medicaid-insured patients compared with matched controls (Figure 1). Pharmacy costs accounted for 44\% of commercial costs (49\% Medicaid) among CPP patients but only 19\% of commercial costs (13\% Medicaid) for controls.

\section{Discussion}

This retrospective cohort study confirms and extends previous studies on the real-world comorbidities, HRU, and costs of GnRH agonist-treated CPP for patients with commercial or Medicaid insurance. Regardless of payer, patients with treated CPP had a higher overall clinical burden, as measured by unique ICD-9-CM diagnostic codes and unique NDC numbers, than matched controls in the 12 months before the index date. Compared with controls, CPP patients experienced higher rates of comorbidities across all evaluated categories, leading to higher pre-index all-cause HRU and costs. Postindex, CPP patients' utilization of services related to disease monitoring was higher than controls, and their mean total 


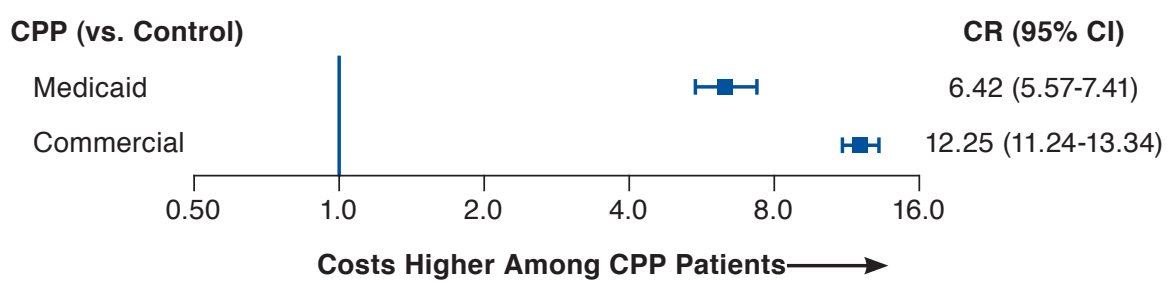

health care costs doubled, driven primarily by outpatient services and outpatient prescriptions. As a proportion of total costs, pharmacy costs increased from $16 \%-21 \%$ of total health care costs in the pre-index period to $44 \%-49 \%$ of total health care costs in the post-index period.

CPP patients with Medicaid insurance had more comorbidities, as measured by unique ICD-9-CM codes and NDC numbers, than commercially insured patients. In addition, Medicaid-insured patients received GnRH agonist treatment for a shorter duration and discontinued treatment at an earlier age than their commercially insured counterparts. Patients with Medicaid insurance were far less likely to visit a pediatric endocrinologist, consistent with other research that has shown significant barriers to specialty care access for patients with public insurance in the United States. ${ }^{28,29}$

This study extends the 2012 study by Chen et al., who used the MarketScan database to evaluate the HRU and costs among a cohort of 172 commercially insured CPP patients compared with matched controls at a ratio of 1:4. ${ }^{23}$ Patients in that study were selected from the period January 1, 2004-June 30, 2006, using criteria similar to those employed in this study. In that analysis, a similarly high prevalence of comorbid conditions, high HRU, and high costs for patients with CPP compared with controls were observed. The mean 12 -month post-index costs (SD) in the Chen et al. study were $\$ 26,819(\$ 43,198)$ for CPP patients and $\$ 1,081(\$ 2,597)$ for controls (adjusted to 2015 U.S. dollars). Within the 2 populations studied, these costs were 12.3 times higher for commercially insured patients and 6.4 times higher for Medicaid-insured patients versus their matched controls. The current study confirms the trends found by Chen et al., extends it to the Medicaid-insured population, and shows more comorbidities, higher HRU, and higher costs in patients with CPP than their non-CPP peers. Further, these results are consistent with the cost of care for other orphan diseases, such as Prader-Willi Syndrome (PWS). In 1 study that investigated the direct medical costs of PWS, such costs per patient per year were 8.8-fold higher and 7.7-fold higher for PWS compared with matched controls for commercial/Medicare-supplemental and Medicaid populations, respectively. ${ }^{30}$

\section{Limitations}

This study is limited by the variables available in the administrative claims databases to fully capture CPP-related treatments, symptoms, and direct medical costs. In particular, over-thecounter medications and other self-management techniques are not tracked. Furthermore, ICD-9-CM diagnostic codes for precocious puberty syndromes are imprecise (although the approach of combining ICD codes, age range, and a specific prescription category is as specific or better than other published studies on (PP). Multivariable analysis was used to control for nonrandom selection into the treatment group; however, there may be residual confounding factors from variables that are not captured in administrative claims data. In addition, results are only representative of the commercially or Medicaid-insured populations, not those who are uninsured or have other insurance. This may particularly affect conclusions about duration of treatment, as it is unknown if these children continued treatment via another payment structure.

\section{Conclusions}

Patients with CPP incur significantly greater HRU and costs than demographically similar non-CPP controls; this finding was present in patients with commercial insurance and Medicaid. In the year leading up to diagnosis, patients with CPP had more comorbidities, higher HRU, and higher costs than their non-CPP peers. These differences remained after diagnosis, with costs continuing to rise as patients began GnRH agonist treatment and long-term management. The substantial economic burden of CPP highlights the need for further evaluation of CPP treatment and management. 


\section{Authors}

KAREN KLEIN, MD, Rady Children's Hospital and University of California, San Diego. AHMED M. SOLIMAN, MS, PhD, and ELIZABETH GRUBB, PhD, MBA, AbbVie, North Chicago, Illinois. MACHAON BONAFEDE, PhD, MPH, IBM Watson Health, Cambridge, Massachusetts, and JAMES K. NELSON, PhD, IBM Watson Health, Ann Arbor, Michigan.

AUTHOR CORRESPONDENCE: Machaon Bonafede, PhD, MPH, 150 Cambridge Park Dr., Cambridge, MA 02140.

Tel.: 603.580.1292; E-mail: mbonafed@us.ibm.com.

\section{DISCLOSURES}

All funding for this study was provided by AbbVie, which participated in analysis and interpretation of data, drafting, reviewing, and approving the publication. All authors contributed to the development of the publication and maintained control over the final content. Soliman and Grubb are employed by AbbVie and hold stock in AbbVie. Bonafede and Nelson are employed by IBM Watson Health, which received funding from AbbVie to conduct this study. Klein is a paid consultant of AbbVie but was not compensated for any work on development of this manuscript for publication.

Portions of this work were presented at Pediatric Academic Societies (PAS) 2018 Meeting, May 5-8, 2018, in Toronto, Canada, as a poster presentation titled "Examination of Economic Burden Among Commercially Insured Patients with Central Precocious Puberty (CPP)."

\section{ACKNOWLEDGMENTS}

Medical writing support was provided by Jessamine P. Winer-Jones, PhD, of IBM Watson Health. These services were paid for by AbbVie.

\section{REFERENCES}

1. Latronico AC, Brito VN, Carel JC. Causes, diagnosis, and treatment of central precocious puberty. Lancet Diabetes Endocrinol. 2016;4(3):265-74.

2. Teilmann G, Pedersen CB, Jensen TK, Skakkebaek NE, Juul A.

Prevalence and incidence of precocious pubertal development in Denmark: an epidemiologic study based on national registries. Pediatrics. 2005;116(6):1323-28.

3. Soriano-Guillén L, Corripio R, Labarta JI, et al. Central precocious puberty in children living in Spain: incidence, prevalence, and influence of adoption and immigration. J Clin Endocrinol Metab. 2010;95(9):4305-13.

4. Sørensen K, Mouritsen A, Aksglaede L, Hagen CP, Mogensen SS, Juul A Recent secular trends in pubertal timing: implications for evaluation and diagnosis of precocious puberty. Horm Res Paediatr. 2012;77(3):137-45.

5. Kaplowitz PB, Oberfield SE. Reexamination of the age limit for defining when puberty is precocious in girls in the United States: implications for evaluation and treatment. Pediatrics. 1999;104(4 Pt 1):936-41.

6. Ng SM, Kumar Y, Cody D, Smith CS, Didi M. Cranial MRI scans are indicated in all girls with central precocious puberty. Arch Dis Child. 2003;88(5):414-18.

7. De Sanctis V, Corrias A, Rizzo V, et al. Etiology of central precocious puberty in males: the results of the Italian Study Group for Physiopathology of Puberty. J Pediatr Endocrinol Metab. 2000;13(Suppl 1):687-93.

8. Pescovitz OH, Comite F, Hench K, et al. The NIH experience with precocious puberty: diagnostic subgroups and response to short-term luteinizing hormone releasing hormone analogue therapy. J Pediatr. 1986;108(1):47-54.
9. Bulcao Macedo D, Nahime Brito V, Latronico AC. New causes of central precocious puberty: the role of genetic factors. Neuroendocrinology. 2014;100(1):1-8.

10. Brito VN, Latronico AC, Arnhold IJ, Mendonca BB. Update on the etiology, diagnosis and therapeutic management of sexual precocity. Arq Bras Endocrinol Metabol. 2008;52(1):18-31.

11. Thamdrup E. Precocious sexual development. A clinical study of 100 children. Obstet Gynecol Surv. 1962;17(3):379-80.

12. Prentice P, Viner RM. Pubertal timing and adult obesity and cardiometabolic risk in women and men: a systematic review and meta-analysis. Int $J$ Obes (Lond). 2013;37(8):1036-43.

13. Lakshman R, Forouhi NG, Sharp SJ, et al. Early age at menarche associated with cardiovascular disease and mortality. J Clin Endocrinol Metab. 2009;94(12):4953-60.

14. Stice E, Presnell K, Bearman SK. Relation of early menarche to depression, eating disorders, substance abuse, and comorbid psychopathology among adolescent girls. Dev Psychol. 2001;37(5):608-19.

15. Franceschi R, Gaudino R, Marcolongo A, et al. Prevalence of polycystic ovary syndrome in young women who had idiopathic central precocious puberty. Fertil Steril. 2010;93(4):1185-91.

16. Williams VSL, Soliman AM, Barret AM, Klein KO. Review and evaluation of patient-centered psychosocial assessments for children with central precocious puberty or early puberty. J Pediatr Endocrinol Metab. 2018;31(5):485-95.

17. Carel JC, Eugster EA, Rogol A, et al. Consensus statement on the use of gonadotropin-releasing hormone analogs in children. Pediatrics. 2009; 123(4):e752-62

18. Eugster EA, Clarke W, Kletter GB, et al. Efficacy and safety of histrelin subdermal implant in children with central precocious puberty: a multicenter trial. J Clin Endocrinol Metab. 2007;92(5):1697-704.

19. Carel JC, Lahlou N, Jaramillo O, et al. Treatment of central precocious puberty by subcutaneous injections of leuprorelin 3-month depot (11.25 mg). J Clin Endocrinol Metab. 2002;87(9):4111-16.

20. Kauli R, Galatzer A, Kornreich L, Lazar L, Pertzelan A, Laron Z. Final height of girls with central precocious puberty, untreated versus treated with cyproterone acetate or GnRH analogue. A comparative study with re-evaluation of predictions by the Bayley-Pinneau method. Horm Res. 1997;47(2):54-61.

21. Lazar L, Lebenthal Y, Yackobovitch-Gavan M, et al. Treated and untreated women with idiopathic precocious puberty: BMI evolution, metabolic outcome, and general health between third and fifth decades. J Clin Endocrinol Metab. 2015;100(4):1445-51.

22. Lazar L, Meyerovitch J, de Vries L, Phillip M, Lebenthal Y. Treated and untreated women with idiopathic precocious puberty: long-term followup and reproductive outcome between the third and fifth decades. Clin Endocrinol (Oxf). 2014;80(4):570-76.

23. Chen SY, Fuldeore M, Boulanger L, Fraser KA, Chwalisz K, Marx SE. Medical resource use and costs related to central precocious puberty: a retrospective cohort study. Endocr Pract. 2012;18(4):519-28.

24. Partsch CJ, Heger S, Sippell WG. Management and outcome of central precocious puberty. Clin Endocrinol (Oxf). 2002;56(2):129-48.

25. Fahmy JL, Kaminsky CK, Kaufman F, Nelson MD, Parisi MT. The radiological approach to precocious puberty. Brit J Radiol. 2000;73(869):560-7.

26. Hankin C, Silverman L, Wang Z, Bronstone A. Association between central precocious puberty and comorbid medical illness: large-scale retrospective claims analysis of Florida Medicaid-enrolled children. Value Health. 2007;10(6):A373.

27. U.S. Department of Labor. Consumer Price Index detailed report: annual average of 2015 tables. Available at: https://www.bls.gov/cpi/tables/detailedreports/home.htm. Accessed May 20, 2019. 
Health Care Utilization and Economic Burden in Patients with Central Precocious Puberty:

An Assessment of the Commercially Insured and Medicaid Populations

28. Skinner AC, Mayer ML. Effects of insurance status on children's access to specialty care: a systematic review of the literature. BMC Health Serv Res. 2007;7(1):194.

29. Bisgaier J, Rhodes KV. Auditing access to specialty care for children with public insurance. N Engl J Med. 2011;364(24):2324-33.

30. Shoffstall AJ, Gaebler JA, Kreher NC, et al. The high direct medical costs of Prader-Willi syndrome. J Pediatr. 2016;175:137-43. 
Health Care Utilization and Economic Burden in Patients with Central Precocious Puberty:

An Assessment of the Commercially Insured and Medicaid Populations

\section{APPENDIX A List of ICD-9-CM Codes Used to Identify Comorbidities and Clinical Characteristics}

Comorbidity and Clinical Characteristics

Endocrine, nutritional, metabolic, and immunity disorders

Hypothyroidism

Diabetes

Obesity

Mental health disorders

Depression

Diseases of the nervous system

Hydrocephalus

Brain tumors

Neurofibromatosis, type 1

Diseases of the musculoskeletal system and connective tissue

Cerebrovascular disease

Renal disease

Chronic pulmonary diseases
ICD-9-CM Codes

204.xx-279.xx

243.xx-244.xx

$250 . x x$

278.xx, V85.2x-4x

290.xx-319.xx

$296.2 x-269.3 x, 311,298$

320.xx-359.xx

$331.3-331.5$

$191 . \mathrm{x}$

237.71

710.xx-739.xx

430.xx-438.xx

430.xx-438.xx, 792.5, 585.6, 996.56, 996.68, 996.73, E879.1, V45.1xx, V56.xx,

585.1-585.5, 403.xx, 404.xx, 249.4, 249.41, 250.4, 250.41, 250.42, 250.43, 285.21,

458.21, 581.xx, 582.xx, 583.xx, 584.xx, 585.9, 586, 588, 588.8x, 588.9

$3.22,6.4,11.4 x, 11.6 x, 20.3-20.5,21.2,22.1,31,39.1,41.3,52.1,55.1,73,79.6,95.1$

$112.4,115.05,115.15,115.95,122.1,130.4,135,306.1,416,466 . x x, 480 . x x, 481$,

482.xx, 483.xx, 484.xx, 485, 486, 487.xx, 488.xx, 490, 491.xx, 494.xx, 495.xx, 496,

500.xx-518.xx, 510.xx-518.xx, 519.8, 519.9, 573.5, 713.4, 714.81, 731.2, 748.4, 748.5

$748.6,748.69,755.54,770,786.0 x, 786.1,786.52,786.9,793.11,793.19,794.2,799.1$

$861.2 \mathrm{x}, 861.3 \mathrm{x}, 997.31,997.32, \mathrm{~V} 12.61, \mathrm{~V} 46.1 \mathrm{x}$ 
Health Care Utilization and Economic Burden in Patients with Central Precocious Puberty:

An Assessment of the Commercially Insured and Medicaid Populations

\section{APPENDIX B Patient Attrition for Commercial and Medicaid Patient Samples}

\section{Commercial Sample}

Patients who have $\geq 1$ claim with a diagnosis of CPP (ICD-9-CM 259.1) from January 1, 2010, through September 30, 2014

$$
\mathrm{N}=80,727
$$

Patients with $\geq 1$ outpatient pharmacy claim for an FDA-approved GnRH for treating CPP on or after their first CPP diagnosis, the earliest of which was the index date

$$
n=3,736
$$

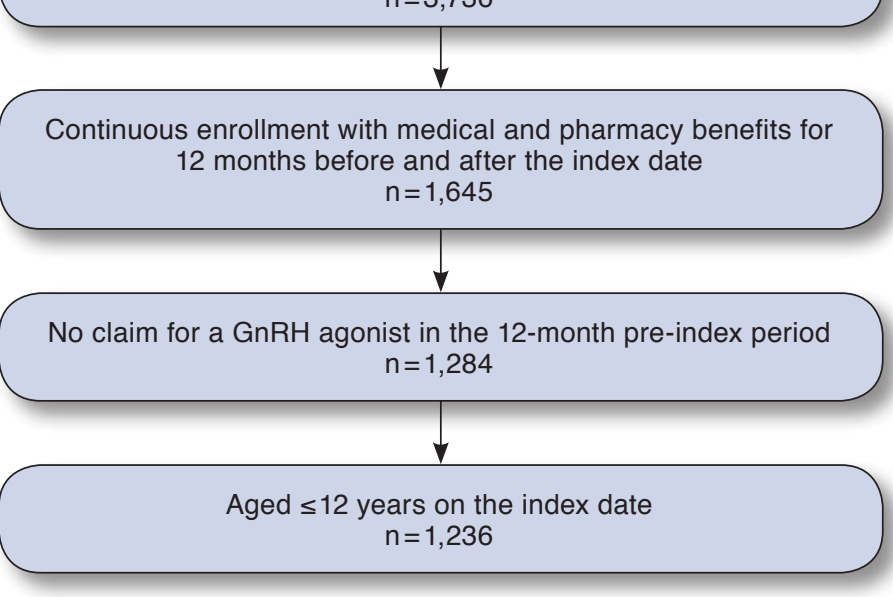

CPP=central precocious puberty; FDA = U.S. Food and Drug Administration; GnRH=gonadotropin-releasing hormone; ICD-9-CM=International Classification of Diseases, Ninth Revision, Clinical Modification.

\section{Medcaid Sample}

Patients who have $\geq 1$ claim with a diagnosis of CPP (ICD-9-CM 259.1) from January 1, 2010, through September 30, 2014

$$
\mathrm{N}=35,242
$$

Patients with at least 1 outpatient pharmacy claim for an FDAapproved GnRH for treating CPP on or after their first CPP

diagnosis, the earliest of which was the index date

$$
n=1,334
$$

Continuous enrollment with medical and pharmacy benefits for 12 months before and after the index date

$$
\mathrm{n}=893
$$

No claim for a GnRH agonist in the 12-month pre-index period $\mathrm{n}=689$

\section{Aged $\leq 12$ years on the index date}

$$
n=673
$$

